\title{
Erratum: Bartram et al (2018)
}

In the article Bartram JC, Thewlis D, Martin DT, Norton KI. Accuracy of $W^{\prime}$ recovery kinetics in high performance cyclists—-modeling intermittent work capacity. Int J Sports Physiol Perform. 2018;13(6):724-728, https://doi.org/10.1123/ijspp.2017-0034, there was a typo in the equation for Skiba 2 in Figure 1-the numerator and denominator of the ending fraction in the last centered equation were transposed. That fraction should have been $W^{\prime} / D_{C P}$, not $D_{C P} / W^{\prime}$. The online version of this article has been corrected. The authors apologize for the error. 